Article

\title{
Intuitionistic Falling Shadow Theory with Applications in BCK/BCI-Algebras
}

\author{
Young Bae Jun ${ }^{1}$, Seok-Zun Song ${ }^{2, *} \mathbb{1}$ and Kyoung Ja Lee ${ }^{3}$ \\ 1 Department of Mathematics Education, Gyeongsang National University, Jinju 52828, Korea; \\ skywine@gmail.com \\ 2 Department of Mathematics, Jeju National University, Jeju 63243, Korea \\ 3 Department of Mathematics Education, Hannam University, Daejeon 306-791, Korea; 1sj01109@gmail.com \\ * Correspondence: szsong@jejunu.ac.kr
}

Received: 24 July 2018; Accepted: 6 August 2018; Published: 13 August 2018

\begin{abstract}
Intuitionistic falling shadow is introduced, and applied to $B C K / B C I$-algebras. Falling intuitionistic subalgebra and falling intuitionistic ideal of $B C K / B C I$-algebras are introduced, and related properties are investigated. Relations between falling intuitionistic subalgebra and falling intuitionistic ideal are discussed. A characterization of falling intuitionistic ideal is established.
\end{abstract}

Keywords: intuitionistic fuzzy subalgebra; intuitionistic fuzzy ideal; intuitionistic random set; intuitionistic falling shadow; falling intuitionistic subalgebra; falling intuitionistic ideal

MSC: 06F35, 03G25, 08A72.

\section{Introduction}

Attanassov [1] introduced the concept of intuitionistic fuzzy set which is a generalization of the fuzzy set. Then, this notion has contributed several branches within the pure and applied sciences. Goodman [2] pointed out the equivalence of a fuzzy set and a class of random sets in the study of a unified treatment of uncertainty modeled by means of combining probability and fuzzy set theory. Wang and Sanchez [3] discussed the theory of falling shadows, and the mathematical structure of the theory of falling shadows is formulated in [4]. Tan et al. [5,6] established a theoretical approach to define a fuzzy inference relation and fuzzy set operations based on the theory of falling shadows. Jun and Park [7] considered a fuzzy subalgebra and a fuzzy ideal as the falling shadow of the cloud of the subalgebra and ideal. Jun and Kang [8] discussed fuzzy positive implicative ideals of $B C K$-algebras based on the theory of falling shadows.

In this manuscript, as a generalization of the concept of fuzzy random set and fuzzy falling shadow, we introduce the notion of intuitionistic random set and intuitionistic falling shadow. Using these notions, we also introduce the concept of falling intuitionistic subalgebra and falling intuitionistic ideal of $B C K / B C I$-algebras, and investigate related properties. We discuss relations between falling intuitionistic subalgebra and falling intuitionistic ideal. We establish a characterization of falling intuitionistic ideal.

\section{Preliminaries}

A $B C K / B C I$-algebra is introduced by $K$. Iséki (see $[9,10]$ ), and it is an important class of logical algebras.

By a $B C K / B C I$-algebra we mean a structure $(X ; *, 0)$ satisfying the following conditions:

(I) $(\forall a, b, c \in X)(((a * b) *(a * c)) *(c * b)=0)$,

(II) $(\forall a, b \in X)((a *(a * b)) * b=0)$, 
(III) $(\forall a \in X)(a * a=0)$,

(IV) $(\forall a, b \in X)(a * b=0, b * a=0 \Rightarrow a=b)$.

If a $B C I$-algebra $X$ satisfies the following identity:

(V) $(\forall a \in X)(0 * a=0)$,

then $X$ is called a $B C K$-algebra. Any $B C K / B C I$-algebra $X$ satisfies the following conditions:

$$
\begin{aligned}
& (\forall a \in X)(a * 0=a), \\
& (\forall a, b, c \in X)(a \leq b \Rightarrow a * c \leq b * c, c * b \leq c * a), \\
& (\forall a, b, c \in X)((a * b) * c=(a * c) * b)
\end{aligned}
$$

where $x \leq y$ if and only if $x * y=0$.

We say that a nonempty subset $S$ in a $B C K / B C I$-algebra $X$ is a subalgebra of $X$ if $a * b \in S$ for all $a, b \in S$. We say that a subset $I$ of a $B C K / B C I$-algebra $X$ is an ideal of $X$ if it satisfies:

$$
\begin{aligned}
& 0 \in I, \\
& (\forall a \in X)(\forall b \in I)(a * b \in I \Rightarrow a \in I) .
\end{aligned}
$$

We refer the reader to the books $[11,12]$ for further information regarding $B C K / B C I$-algebras.

An intuitionistic fuzzy set $f=\left(f_{\alpha}, f_{\beta}\right)$ in a $B C K / B C I$-algebra $X$ is called an intuitionistic fuzzy subalgebra of $X$ (see [13]) if it satisfies:

$$
(\forall a, b \in X)\left(\begin{array}{l}
f_{\alpha}(a * b) \geq \min \left\{f_{\alpha}(a), f_{\alpha}(b)\right\} \\
f_{\beta}(a * b) \leq \max \left\{f_{\beta}(a), f_{\beta}(b)\right\}
\end{array}\right) .
$$

An intuitionistic fuzzy set $f=\left(f_{\alpha}, f_{\beta}\right)$ in a $B C K / B C I$-algebra $X$ is called an intuitionistic fuzzy ideal of $X$ (see [13]) if it satisfies:

$$
\begin{gathered}
(\forall y \in X)\left(f_{\alpha}(0) \geq f_{\alpha}(y), f_{\beta}(0) \leq f_{\beta}(y)\right) . \\
(\forall a, b \in X)\left(\begin{array}{l}
f_{\alpha}(a) \geq \min \left\{f_{\alpha}(a * b), f_{\alpha}(b)\right\} \\
f_{\beta}(a) \leq \max \left\{f_{\beta}(a * b), f_{\beta}(b)\right\}
\end{array}\right) .
\end{gathered}
$$

For any $\left(t_{\alpha}, t_{\beta}\right) \in[0,1] \times[0,1]$ and an intuitionistic fuzzy set $f=\left(f_{\alpha}, f_{\beta}\right)$ in a $B C K / B C I$-algebra $X$, consider the following sets:

$$
U\left(f ; t_{\alpha}\right)=\left\{x \in X \mid f_{\alpha}(x) \geq t_{\alpha}\right\}
$$

and

$$
L\left(f ; t_{\beta}\right)=\left\{x \in X \mid f_{\beta}(x) \leq t_{\beta}\right\} .
$$

\section{Intuitionistic Fuzzification of Subalgebras/Ideals Based on Intuitionistic Falling Shadows}

Given a $B C K / B C I$-algebra $X, x \in X$ and $D \in 2^{X}$, let

$$
\bar{x}:=\left\{C \in 2^{X} \mid x \in C\right\},
$$

and

$$
\bar{D}:=\{\bar{x} \mid x \in D\} .
$$


An ordered pair $\left(2^{X}, \mathcal{B}\right)$ is said to be a hyper-measurable structure on $X$ if $\mathcal{B}$ is a $\sigma$-field in $2^{X}$ and $\bar{X} \subseteq \mathcal{B}$.

Given a probability space $(\Gamma, \mathcal{A}, P)$ and a hyper-measurable structure $\left(2^{X}, \mathcal{B}\right)$ on $X$, an intuitionistic random set on $X$ is defined to be a couple $\zeta:=\left(\zeta_{\alpha}, \zeta_{\beta}\right)$ in which $\zeta_{\alpha}$ and $\zeta_{\beta}$ are mappings from $\Gamma$ to $2^{X}$ which are $\mathcal{A}-\mathcal{B}$ measurables, that is,

$$
(\forall C \in \mathcal{B})\left(\begin{array}{c}
\zeta_{\alpha}^{-1}(C)=\left\{\omega_{\alpha} \in \Gamma \mid \zeta_{\alpha}\left(\omega_{\alpha}\right) \in C\right\} \in \mathcal{A} \\
\zeta_{\beta}^{-1}(C)=\left\{\omega_{\beta} \in \Gamma \mid \zeta_{\beta}\left(\omega_{\beta}\right) \in C\right\} \in \mathcal{A}
\end{array}\right) .
$$

Given an intuitionistic random set $\zeta:=\left(\zeta_{\alpha}, \zeta_{\beta}\right)$ on $X$, consider functions:

$$
\begin{aligned}
& \tilde{G}_{\alpha}: X \rightarrow[0,1], x_{\alpha} \mapsto P\left(\omega_{\alpha} \mid x_{\alpha} \in \zeta_{\alpha}\left(\omega_{\alpha}\right)\right), \\
& \tilde{G}_{\beta}: X \rightarrow[0,1], x_{\beta} \mapsto 1-P\left(\omega_{\beta} \mid x_{\beta} \in \zeta_{\beta}\left(\omega_{\beta}\right)\right) .
\end{aligned}
$$

Then $\tilde{G}:=\left(\tilde{G}_{\alpha}, \tilde{G}_{\beta}\right)$ is an intuitionistic fuzzy set on $X$, and we call it the intuitionistic falling shadow of the intuitionistic random set $\zeta:=\left(\zeta_{\alpha}, \zeta_{\beta}\right)$, and $\zeta:=\left(\zeta_{\alpha}, \zeta_{\beta}\right)$ is called a intuitionistic cloud of $\tilde{G}:=\left(\tilde{G}_{\alpha}, \tilde{G}_{\beta}\right)$.

For example, consider a probability space $(\Gamma, \mathcal{A}, P)=([0,1], \mathcal{A}, m)$ where $\mathcal{A}$ is a Borel field on $[0,1]$ and $m$ is the usual Lebesgue measure. Let $\tilde{G}:=\left(\tilde{G}_{\alpha}, \tilde{G}_{\beta}\right)$ be an intuitionistic fuzzy set in $X$. Then a couple $\zeta:=\left(\zeta_{\alpha}, \zeta_{\beta}\right)$ in which

$$
\begin{aligned}
& \zeta_{\alpha}:[0,1] \rightarrow 2^{X}, t_{\alpha} \mapsto U\left(\tilde{G} ; t_{\alpha}\right), \\
& \zeta_{\beta}:[0,1] \rightarrow 2^{X}, t_{\beta} \mapsto L\left(\tilde{G} ; t_{\beta}\right)
\end{aligned}
$$

is an intuitionistic random set and $\zeta:=\left(\zeta_{\alpha}, \zeta_{\beta}\right)$ is an intuitionistic cloud of $\tilde{G}:=\left(\tilde{G}_{\alpha}, \tilde{G}_{\beta}\right)$. We will call $\zeta:=\left(\zeta_{\alpha}, \zeta_{\beta}\right)$ defined above as the intuitionistic cut-cloud of $\tilde{G}:=\left(\tilde{G}_{\alpha}, \tilde{G}_{\beta}\right)$.

Definition 1. Let $(\Gamma, \mathcal{A}, P)$ be a probability space and let $\zeta:=\left(\zeta_{\alpha}, \zeta_{\beta}\right)$ be an intuitionistic random set on a BCK/BCI-algebra X. Then the intuitionistic falling shadow $\tilde{G}:=\left(\tilde{G}_{\alpha}, \tilde{G}_{\beta}\right)$ of $\zeta:=\left(\zeta_{\alpha}, \zeta_{\beta}\right)$ is called a falling intuitionistic subalgebra (resp., falling intuitionistic ideal) of $X$ if $\zeta_{\alpha}\left(\omega_{\alpha}\right)$ and $\zeta_{\beta}\left(\omega_{\beta}\right)$ are subalgebras (resp., ideals) of $X$ for all $\omega_{\alpha}, \omega_{\beta} \in \Gamma$.

Example 1. Consider a set $X=\{0,1,2,3,4\}$ with the binary operation $*$ which is given in Table 1.

Table 1. Cayley table for the binary operation " $*$ ".

\begin{tabular}{lllllll}
\hline$*$ & 0 & 1 & 2 & 3 & 4 \\
\hline 0 & 0 & 0 & 0 & 0 & 0 \\
1 & 1 & 0 & 1 & 0 & 0 \\
2 & 2 & 2 & 0 & 0 & 0 \\
3 & 3 & 3 & 3 & 0 & 0 \\
4 & 4 & 3 & 4 & 1 & 0 \\
\hline
\end{tabular}

Then $(X ; *, 0)$ is a BCK-algebra (see [12]). Let $\zeta:=\left(\zeta_{\alpha}, \zeta_{\beta}\right)$ be an intuitionistic random set on $X$ which is given as follows:

$$
\zeta_{\alpha}:[0,1] \rightarrow 2^{X}, \quad x \mapsto \begin{cases}\{0,1\} & \text { if } t \in[0,0.3) \\ \{0,2\} & \text { if } t \in[0.3,0.7) \\ \{0,1,2\} & \text { if } t \in[0.7,0.8) \\ X & \text { if } t \in[0.8,1]\end{cases}
$$


and

$$
\zeta_{\beta}:[0,1] \rightarrow 2^{X}, \quad x \mapsto \begin{cases}\{0\} & \text { if } t \in(0.9,1] \\ \{0,1\} & \text { if } t \in(0.7,0.9] \\ \{0,2\} & \text { if } t \in(0.5,0.7] \\ \{0,1,2\} & \text { if } t \in(0.3,0.5] \\ X & \text { if } t \in[0,0.3] .\end{cases}
$$

Then $\zeta_{\alpha}(t)$ and $\zeta_{\beta}(t)$ are subalgebras and ideals of $X$ for all $t \in[0,1]$. Hence the intuitionistic falling shadow $\tilde{G}:=\left(\tilde{G}_{\alpha}, \tilde{G}_{\beta}\right)$ of $\zeta:=\left(\zeta_{\alpha}, \zeta_{\beta}\right)$ is a falling intuitionistic subalgebra and a falling intuitionistic ideal of $X$, and it is given as follows:

$$
\begin{aligned}
& \tilde{G}_{\alpha}(x)= \begin{cases}1 & \text { if } x=0, \\
0.6 & \text { if } x=1, \\
0.7 & \text { if } x=2, \\
0.2 & \text { if } x \in\{3,4\},\end{cases} \\
& \tilde{G}_{\beta}(x)= \begin{cases}0 & \text { if } x=0, \\
0.3 & \text { if } x \in\{1,2\}, \\
0.7 & \text { if } x \in\{3,4\} .\end{cases}
\end{aligned}
$$

Let $X$ be a $B C K / B C I$-algebra. Given a probability space $(\Gamma, \mathcal{A}, P)$, let

$$
\mathcal{F}(X):=\{f \mid f: \Gamma \rightarrow X \text { is a mapping }\} .
$$

Define a binary operation $\circledast$ on $\mathcal{F}(X)$ as follows:

$$
(\forall \omega \in \Gamma)((f \circledast g)(\omega)=f(\omega) * g(\omega))
$$

for all $f, g \in \mathcal{F}(X)$. Then $(\mathcal{F}(X) ; \circledast, \mathbf{0})$ is a $B C K / B C I$-algebra (see [7]) where $\mathbf{0}$ is given as follows:

$$
\mathbf{0}: \Gamma \rightarrow X, \omega \mapsto 0 .
$$

For any subset $A$ of a $B C K / B C I$-algebra $X$ and $g_{\alpha}, g_{\beta} \in \mathcal{F}(X)$, consider the following sets:

$$
\begin{aligned}
& A_{\alpha}^{g}:=\left\{\omega_{\alpha} \in \Gamma \mid g_{\alpha}\left(\omega_{\alpha}\right) \in A\right\}, \\
& A_{\beta}^{g}:=\left\{\omega_{\beta} \in \Gamma \mid g_{\beta}\left(\omega_{\beta}\right) \in A\right\}
\end{aligned}
$$

and

$$
\begin{aligned}
& \zeta_{\alpha}: \Gamma \rightarrow 2^{\mathcal{F}(X)}, \omega_{\alpha} \mapsto\left\{g_{\alpha} \in \mathcal{F}(X) \mid g_{\alpha}\left(\omega_{\alpha}\right) \in A\right\}, \\
& \zeta_{\beta}: \Gamma \rightarrow 2^{\mathcal{F}(X)}, \omega_{\beta} \mapsto\left\{g_{\beta} \in \mathcal{F}(X) \mid g_{\beta}\left(\omega_{\beta}\right) \in A\right\} .
\end{aligned}
$$

Then $A_{\alpha}^{g}, A_{\beta}^{g} \in \mathcal{A}$.

Assume that $A$ is a subalgebra (resp., ideal) of a $B C K / B C I$-algebra $X$ and let $\omega_{\alpha}, \omega_{\beta} \in \Gamma$. Since $\mathbf{0}(\omega)=0 \in A$ for $\omega \in\left\{\omega_{\alpha}, \omega_{\beta}\right\}$, we know that $\mathbf{0} \in \zeta_{\alpha}\left(\omega_{\alpha}\right)$ and $\mathbf{0} \in \zeta_{\beta}\left(\omega_{\beta}\right)$. For any $f_{\alpha}$, $g_{\alpha} \in \mathcal{F}(X)$, if $f_{\alpha}, g_{\alpha} \in \zeta_{\alpha}\left(\omega_{\alpha}\right)$, then

$$
\left(f_{\alpha} \circledast g_{\alpha}\right)\left(\omega_{\alpha}\right)=f_{\alpha}\left(\omega_{\alpha}\right) * g_{\alpha}\left(\omega_{\alpha}\right) \in A
$$

and so $f_{\alpha} \circledast g_{\alpha} \in \zeta_{\alpha}\left(\omega_{\alpha}\right)$. Thus $\zeta_{\alpha}\left(\omega_{\alpha}\right)$ is a subalgebra of $\mathcal{F}(X)$ for all $\omega_{\alpha} \in \Gamma$. If $f_{\alpha} \circledast g_{\alpha} \in \zeta_{\alpha}\left(\omega_{\alpha}\right)$ and $g_{\alpha} \in \zeta_{\alpha}\left(\omega_{\alpha}\right)$, then $f_{\alpha}\left(\omega_{\alpha}\right) * g_{\alpha}\left(\omega_{\alpha}\right)=\left(f_{\alpha} \circledast g_{\alpha}\right)\left(\omega_{\alpha}\right) \in A$ and $g_{\alpha}\left(\omega_{\alpha}\right) \in A$. Since $A$ is an ideal of $X$, 
it follows that $f_{\alpha}\left(\omega_{\alpha}\right) \in A$, i.e., $f_{\alpha} \in \zeta_{\alpha}\left(\omega_{\alpha}\right)$. Hence $\zeta_{\alpha}\left(\omega_{\alpha}\right)$ is an ideal of $\mathcal{F}(X)$ for all $\omega_{\alpha} \in \Gamma$. By the similar way, we can verify that $\zeta_{\beta}\left(\omega_{\beta}\right)$ is a subalgebra (resp., ideal) of $\mathcal{F}(X)$ for all $\omega_{\beta} \in \Gamma$. Since

$$
\begin{aligned}
& \zeta_{\alpha}^{-1}\left(\bar{g}_{\alpha}\right)=\left\{\omega_{\alpha} \in \Gamma \mid g_{\alpha} \in \zeta_{\alpha}\left(\omega_{\alpha}\right)\right\}=\left\{\omega_{\alpha} \in \Gamma \mid g_{\alpha}\left(\omega_{\alpha}\right) \in A\right\}=A_{\alpha}^{g} \in \mathcal{A}, \\
& \zeta_{\beta}^{-1}\left(\bar{g}_{\beta}\right)=\left\{\omega_{\beta} \in \Gamma \mid g_{\beta} \in \zeta_{\beta}\left(\omega_{\beta}\right)\right\}=\left\{\omega_{\beta} \in \Gamma \mid g_{\beta}\left(\omega_{\beta}\right) \in A\right\}=A_{\beta}^{g} \in \mathcal{A},
\end{aligned}
$$

$\zeta:=\left(\zeta_{\alpha}, \zeta_{\beta}\right)$ is an intuitionistic random set on $\mathcal{F}(X)$. Hence $\tilde{G}:=\left(\tilde{G}_{\alpha}, \tilde{G}_{\beta}\right)$ is a falling intuitionistic subalgebra and a falling intuitionistic ideal of $\mathcal{F}(X)$ where

$$
\begin{aligned}
& \tilde{G}_{\alpha}: \mathcal{F}(X) \rightarrow[0,1], g_{\alpha} \mapsto P\left(\omega_{\alpha} \mid g_{\alpha}\left(\omega_{\alpha}\right) \in A\right), \\
& \tilde{G}_{\alpha}: \mathcal{F}(X) \rightarrow[0,1], g_{\beta} \mapsto 1-P\left(\omega_{\beta} \mid g_{\beta}\left(\omega_{\beta}\right) \in A\right) .
\end{aligned}
$$

Given a probability space $(\Gamma, \mathcal{A}, P)$, let $\tilde{G}:=\left(\tilde{G}_{\alpha}, \tilde{G}_{\beta}\right)$ be an intuitionistic falling shadow of an intuitionistic random set $\zeta:=\left(\zeta_{\alpha}, \zeta_{\beta}\right)$. For an element $x$ of a $B C K / B C I$-algebra $X$, let

$$
\begin{aligned}
& \Gamma\left(x ; \zeta_{\alpha}\right):=\left\{\omega_{\alpha} \in \Gamma \mid x \in \zeta_{\alpha}\left(\omega_{\alpha}\right)\right\} \\
& \Gamma\left(x ; \zeta_{\beta}\right):=\left\{\omega_{\beta} \in \Gamma \mid x \in \zeta_{\beta}\left(\omega_{\beta}\right)\right\} .
\end{aligned}
$$

Then $\Gamma\left(x ; \zeta_{\alpha}\right) \in \mathcal{A}$ and $\Gamma\left(x ; \zeta_{\beta}\right) \in \mathcal{A}$.

Proposition 1. Let $\tilde{G}:=\left(\tilde{G}_{\alpha}, \tilde{G}_{\beta}\right)$ be an intuitionistic falling shadow of the intuitionistic random set $\zeta:=\left(\zeta_{\alpha}, \zeta_{\beta}\right)$. If $\tilde{G}:=\left(\tilde{G}_{\alpha}, \tilde{G}_{\beta}\right)$ is a falling intuitionistic subalgebra of a BCK/BCI-algebra $X$, then

$$
(\forall x, y \in X)\left(\begin{array}{l}
\Gamma\left(x ; \zeta_{\alpha}\right) \cap \Gamma\left(y ; \zeta_{\alpha}\right) \subseteq \Gamma\left(x * y ; \zeta_{\alpha}\right) \\
\Gamma\left(x ; \zeta_{\beta}\right) \cap \Gamma\left(y ; \zeta_{\beta}\right) \subseteq \Gamma\left(x * y ; \zeta_{\beta}\right)
\end{array}\right)
$$

If $\tilde{G}:=\left(\tilde{G}_{\alpha}, \tilde{G}_{\beta}\right)$ is a falling intuitionistic ideal of a BCK/BCI-algebra X, then

$$
\begin{aligned}
& (\forall x, y \in X)\left(x \leq y \Rightarrow\left\{\begin{array}{l}
\Gamma\left(y ; \zeta_{\alpha}\right) \subseteq \Gamma\left(x ; \zeta_{\alpha}\right) \\
\Gamma\left(y ; \zeta_{\beta}\right) \subseteq \Gamma\left(x ; \zeta_{\beta}\right)
\end{array}\right),\right. \\
& (\forall x, y \in X)\left(\begin{array}{l}
\Gamma\left(x * y ; \zeta_{\alpha}\right) \cap \Gamma\left(y ; \zeta_{\alpha}\right) \subseteq \Gamma\left(x ; \zeta_{\alpha}\right) \\
\Gamma\left(x * y ; \zeta_{\beta}\right) \cap \Gamma\left(y ; \zeta_{\beta}\right) \subseteq \Gamma\left(x ; \zeta_{\beta}\right)
\end{array}\right) .
\end{aligned}
$$

If $\tilde{G}:=\left(\tilde{G}_{\alpha}, \tilde{G}_{\beta}\right)$ is a falling intuitionistic ideal/subalgebra of a BCK-algebra X, then

$$
(\forall x \in X)\left(\begin{array}{c}
\Gamma\left(x ; \zeta_{\alpha}\right) \subseteq \Gamma\left(0 ; \zeta_{\alpha}\right) \\
\Gamma\left(x ; \zeta_{\beta}\right) \subseteq \Gamma\left(0 ; \zeta_{\beta}\right)
\end{array}\right) .
$$

If $\tilde{G}:=\left(\tilde{G}_{\alpha}, \tilde{G}_{\beta}\right)$ is a falling intuitionistic ideal of a BCK-algebra $X$, then

$$
(\forall x, y \in X)\left(\begin{array}{c}
\Gamma\left(x ; \zeta_{\alpha}\right) \subseteq \Gamma\left(x * y ; \zeta_{\alpha}\right) \\
\Gamma\left(x ; \zeta_{\beta}\right) \subseteq \Gamma\left(x * y ; \zeta_{\beta}\right)
\end{array}\right) .
$$

Proof. Assume that $\tilde{G}:=\left(\tilde{G}_{\alpha}, \tilde{G}_{\beta}\right)$ is a falling intuitionistic subalgebra of $X$. If $\omega_{\alpha} \in \Gamma\left(x ; \zeta_{\alpha}\right) \cap \Gamma\left(y ; \zeta_{\alpha}\right)$ for any $x, y \in X$, then $x \in \zeta_{\alpha}\left(\omega_{\alpha}\right)$ and $y \in \zeta_{\alpha}\left(\omega_{\alpha}\right)$. Since $\zeta_{\alpha}\left(\omega_{\alpha}\right)$ is a subalgebra of $X$, it follows that $x * y \in \zeta_{\alpha}\left(\omega_{\alpha}\right)$, that is, $\omega_{\alpha} \in \Gamma\left(x * y ; \zeta_{\alpha}\right)$. Now let $\omega_{\beta} \in \Gamma\left(x ; \zeta_{\beta}\right) \cap \Gamma\left(y ; \zeta_{\beta}\right)$ for any $x, y \in X$. Then $x \in \zeta_{\beta}\left(\omega_{\beta}\right)$ and $y \in \zeta_{\beta}\left(\omega_{\beta}\right)$, which imply that $x * y \in \zeta_{\beta}\left(\omega_{\beta}\right)$ since $\zeta_{\beta}\left(\omega_{\beta}\right)$ is a subalgebra

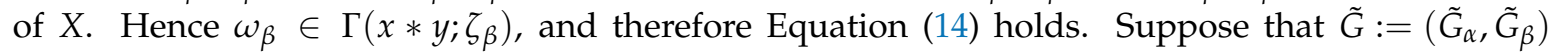
is a falling intuitionistic ideal of $X$ and let $x, y \in X$ be such that $x \leq y$. Then $x * y=0$. If $\omega_{\alpha} \in \Gamma\left(y ; \zeta_{\alpha}\right)$, then $y \in \zeta_{\alpha}\left(\omega_{\alpha}\right)$ and $x * y=0 \in \zeta_{\alpha}\left(\omega_{\alpha}\right)$. Thus $x \in \zeta_{\alpha}\left(\omega_{\alpha}\right)$ since $\zeta_{\alpha}\left(\omega_{\alpha}\right)$ is an ideal 
of $X$. Hence $\omega_{\alpha} \in \Gamma\left(x ; \zeta_{\alpha}\right)$, and so $\Gamma\left(y ; \zeta_{\alpha}\right) \subseteq \Gamma\left(x ; \zeta_{\alpha}\right)$. Let $\omega_{\beta} \in \Gamma\left(y ; \zeta_{\beta}\right)$. Then $y \in \zeta_{\beta}\left(\omega_{\beta}\right)$ and $x * y=0 \in \zeta_{\beta}\left(\omega_{\beta}\right)$, which imply that $x \in \zeta_{\beta}\left(\omega_{\beta}\right)$ since $\zeta_{\beta}\left(\omega_{\beta}\right)$ is an ideal of $X$. Hence $\omega_{\beta} \in \Gamma\left(x ; \zeta_{\beta}\right)$ which shows that $\Gamma\left(y ; \zeta_{\beta}\right) \subseteq \Gamma\left(x ; \zeta_{\beta}\right)$. The inclusions $\Gamma\left(x * y ; \zeta_{\alpha}\right) \cap \Gamma\left(y ; \zeta_{\alpha}\right) \subseteq \Gamma\left(x ; \zeta_{\alpha}\right)$ and $\Gamma\left(x * y ; \zeta_{\beta}\right) \cap \Gamma\left(y ; \zeta_{\beta}\right) \subseteq \Gamma\left(x ; \zeta_{\beta}\right)$ are obtained by the similarly way. Please note that $0 \leq x$ and $x * y \leq x$ in a $B C K$-algebra. Hence the result Equation (15) induces Equations (17) and (18).

Theorem 1. Let $X$ be a $B C K / B C I$-algebra. If we take a probability space $(\Gamma, \mathcal{A}, P)=([0,1], \mathcal{A}, m)$, then every intuitionistic fuzzy subalgebra (resp., intuitionistic fuzzy ideal) of $X$ is a falling intuitionistic subalgebra (resp., falling intuitionistic ideal) of $X$.

Proof. Let $\tilde{G}:=\left(\tilde{G}_{\alpha}, \tilde{G}_{\beta}\right)$ be an intuitionistic fuzzy subalgebra (resp., intuitionistic fuzzy ideal) of a $B C K / B C I$-algebra $X$. Then $U\left(\tilde{G} ; t_{\alpha}\right)$ and $L\left(\tilde{G} ; t_{\beta}\right)$ are subalgebras (resp., ideals) of $X$ for all $\left(t_{\alpha}, t_{\beta}\right) \in[0,1] \times[0,1]$. Hence a couple $\zeta:=\left(\zeta_{\alpha}, \zeta_{\beta}\right)$ in which

$$
\begin{aligned}
& \zeta_{\alpha}:[0,1] \rightarrow 2^{X}, t_{\alpha} \mapsto U\left(\tilde{G} ; t_{\alpha}\right), \\
& \zeta_{\beta}:[0,1] \rightarrow 2^{X}, t_{\beta} \mapsto L\left(\tilde{G} ; t_{\beta}\right)
\end{aligned}
$$

is an intuitionistic cut-cloud of $\tilde{G}:=\left(\tilde{G}_{\alpha}, \tilde{G}_{\beta}\right)$, and so $\tilde{G}:=\left(\tilde{G}_{\alpha}, \tilde{G}_{\beta}\right)$ is a falling intuitionistic subalgebra (resp., falling intuitionistic ideal) of $X$.

The converse of Theorem 1 is not true as seen in the following example.

Example 2. Consider $a$ set $X=\{0, a, b, c\}$ with the binary operation $*$ which is given in Table 2.

Table 2. Cayley table for the binary operation " $*$ ".

\begin{tabular}{lllll}
\hline$*$ & $\mathbf{0}$ & $\mathbf{a}$ & $\mathbf{b}$ & $\mathbf{c}$ \\
\hline 0 & 0 & $a$ & $b$ & $c$ \\
$a$ & $a$ & 0 & $c$ & $b$ \\
$b$ & $b$ & $c$ & 0 & $a$ \\
$c$ & $c$ & $b$ & $a$ & 0 \\
\hline
\end{tabular}

Then $(X ; *, 0)$ is a BCI-algebra (see [12]). Consider $(\Gamma, \mathcal{A}, P)=([0,1], \mathcal{A}, m)$ as a probability space, and let $\zeta:=\left(\zeta_{\alpha}, \zeta_{\beta}\right)$ be an intuitionistic random set on $X$ which is given as follows:

$$
\zeta_{\alpha}:[0,1] \rightarrow 2^{X}, \quad x \mapsto \begin{cases}\{0\} & \text { if } t \in[0,0.2), \\ \{0, a\} & \text { if } t \in[0.2,0.7), \\ \{0, b\} & \text { if } t \in[0.7,0.8), \\ X & \text { if } t \in[0.8,1],\end{cases}
$$

and

$$
\zeta_{\beta}:[0,1] \rightarrow 2^{X}, x \mapsto \begin{cases}\{0\} & \text { if } t \in(0.8,1] \\ \{0, a\} & \text { if } t \in(0.7,0.8] \\ \{0, b\} & \text { if } t \in(0.5,0.7] \\ \{0, c\} & \text { if } t \in[0,0.5] .\end{cases}
$$


Then $\zeta_{\alpha}(t)$ and $\zeta_{\beta}(t)$ are subalgebras of $X$ for all $t \in[0,1]$. Hence the intuitionistic falling shadow $\tilde{G}:=\left(\tilde{G}_{\alpha}, \tilde{G}_{\beta}\right)$ of $\zeta:=\left(\zeta_{\alpha}, \zeta_{\beta}\right)$ is a falling intuitionistic subalgebra of $X$, and it is given as follows:

$$
\tilde{G}_{\alpha}(x)= \begin{cases}1 & \text { if } x=0 \\ 0.7 & \text { if } x=a \\ 0.3 & \text { if } x=b \\ 0.2 & \text { if } x=c\end{cases}
$$

and

$$
\tilde{G}_{\beta}(x)= \begin{cases}0 & \text { if } x=0 \\ 0.9 & \text { if } x=a \\ 0.8 & \text { if } x=b \\ 0.5 & \text { if } x=c\end{cases}
$$

Since $\tilde{G}_{\beta}(b * c)=\tilde{G}_{\beta}(a)=0.9>0.8=\max \left\{\tilde{G}_{\beta}(b), \tilde{G}_{\beta}(c)\right\}$, we know that $\tilde{G}:=\left(\tilde{G}_{\alpha}, \tilde{G}_{\beta}\right)$ is not an intuitionistic fuzzy subalgebra of $X$.

If we take an intuitionistic random set $\zeta:=\left(\zeta_{\alpha}, \zeta_{\beta}\right)$ on $X$ as follows:

$$
\zeta_{\alpha}:[0,1] \rightarrow 2^{X}, x \mapsto \begin{cases}\{0, a\} & \text { if } t \in[0,0.3), \\ \{0, b\} & \text { if } t \in[0.3,0.5), \\ \{0, c\} & \text { if } t \in[0.5,1]\end{cases}
$$

and

$$
\zeta_{\beta}:[0,1] \rightarrow 2^{X}, x \mapsto \begin{cases}\{0\} & \text { if } t \in(0.8,1] \\ \{0, a\} & \text { if } t \in(0.7,0.8] \\ \{0, b\} & \text { if } t \in(0.5,0.7] \\ \{0, c\} & \text { if } t \in[0,0.5] .\end{cases}
$$

Then $\zeta_{\alpha}(t)$ and $\zeta_{\beta}(t)$ are ideals of $X$ for all $t \in[0,1]$. Hence the intuitionistic falling shadow $\tilde{G}:=\left(\tilde{G}_{\alpha}, \tilde{G}_{\beta}\right)$ of $\zeta:=\left(\zeta_{\alpha}, \zeta_{\beta}\right)$ is a falling intuitionistic ideal of $X$, and it is given as follows:

$$
\tilde{G}_{\alpha}(x)= \begin{cases}1 & \text { if } x=0 \\ 0.3 & \text { if } x=a \\ 0.2 & \text { if } x=b \\ 0.5 & \text { if } x=c\end{cases}
$$

and

$$
\tilde{G}_{\beta}(x)= \begin{cases}0 & \text { if } x=0 \\ 0.9 & \text { if } x=a \\ 0.8 & \text { if } x=b \\ 0.5 & \text { if } x=c\end{cases}
$$

Since $\tilde{G}_{\alpha}(b)=0.2<0.3=\min \left\{\tilde{G}_{\alpha}(b * a), \tilde{G}_{\alpha}(a)\right\}$, we know that $\tilde{G}:=\left(\tilde{G}_{\alpha}, \tilde{G}_{\beta}\right)$ is not an intuitionistic fuzzy ideal of $X$.

Theorem 2. If we take a probability space $(\Gamma, \mathcal{A}, P)=([0,1], \mathcal{A}, m)$, then every falling intuitionistic ideal is a falling intuitionistic subalgebra in a BCK-algebra.

Proof. Since every ideal is a subalgebra in a $B C K$-algebra, it is straightforward.

The following example shows that Theorem 2 is not true in a BCI-algebra. 
Example 3. Let $X$ be the set of all nonzero rational numbers. If we take a binary operation $*$ on $X$ defined by division as general, then $(X ; *, 1)$ is a BCI-algebra (see [14]). Consider $(\Gamma, \mathcal{A}, P)=([0,1], \mathcal{A}, m)$ as a probability space, and let $\zeta:=\left(\zeta_{\alpha}, \zeta_{\beta}\right)$ be an intuitionistic random set on $X$ which is given as follows:

$$
\zeta_{\alpha}:[0,1] \rightarrow 2^{X}, x \mapsto \begin{cases}X & \text { if } t \in(0.6,1] \\ \mathbb{Z}^{*} & \text { if } t \in[0,0.6]\end{cases}
$$

and

$$
\zeta_{\beta}:[0,1] \rightarrow 2^{X}, x \mapsto \begin{cases}X & \text { if } t \in[0,0.7), \\ \mathbb{Z}^{*} & \text { if } t \in[0.7,1],\end{cases}
$$

where $\mathbb{Z}^{*}$ is the set of all nonzero integers. Then the intuitionistic falling shadow $\tilde{G}:=\left(\tilde{G}_{\alpha}, \tilde{G}_{\beta}\right)$ of $\zeta:=\left(\zeta_{\alpha}, \zeta_{\beta}\right)$ is a falling intuitionistic ideal of $X$, but it is not a falling intuitionistic subalgebra of $X$ because $\zeta_{\alpha}(0.4)=\mathbb{Z}^{*}$ and/or $\zeta_{\beta}(0.85)=\mathbb{Z}^{*}$ are not subalgebras of $X$ since $2 \in \mathbb{Z}^{*}$ and $3 \in \mathbb{Z}^{*}$ but $2 * 3 \notin \mathbb{Z}^{*}$.

We provide conditions for a falling intuitionistic subalgebra to be a falling intuitionistic ideal in BCI-algebras.

Theorem 3. Given a BCI-algebra $X$, assume that the intuitionistic falling shadow $\tilde{G}:=\left(\tilde{G}_{\alpha}, \tilde{G}_{\beta}\right)$ of an intuitionistic random set $\zeta:=\left(\zeta_{\alpha}, \zeta_{\beta}\right)$ is a falling intuitionistic subalgebra of $X$. Then $\tilde{G}:=\left(\tilde{G}_{\alpha}, \tilde{G}_{\beta}\right)$ is a falling intuitionistic ideal of $X$ if and only if for every $x, y \in X$ and $\omega_{\alpha}, \omega_{\beta} \in \Gamma$, the following conditions are valid:

$$
\begin{aligned}
& x \in \zeta_{\alpha}\left(\omega_{\alpha}\right), y \notin \zeta_{\alpha}\left(\omega_{\alpha}\right) \Rightarrow y * x \notin \zeta_{\alpha}\left(\omega_{\alpha}\right), \\
& x \in \zeta_{\beta}\left(\omega_{\beta}\right), y \notin \zeta_{\alpha}\left(\omega_{\beta}\right) \Rightarrow y * x \notin \zeta_{\beta}\left(\omega_{\beta}\right) .
\end{aligned}
$$

Proof. If $\tilde{G}:=\left(\tilde{G}_{\alpha}, \tilde{G}_{\beta}\right)$ is a falling intuitionistic ideal of a $B C I$-algebra $X$, then $\zeta_{\alpha}\left(\omega_{\alpha}\right)$ and $\zeta_{\beta}\left(\omega_{\beta}\right)$ are ideals of $X$ for all $\omega_{\alpha}, \omega_{\beta} \in \Gamma$. Let $x, y \in X$ be such that $x \in \zeta_{\alpha}\left(\omega_{\alpha}\right)$ and $y \notin \zeta_{\alpha}\left(\omega_{\alpha}\right)$. If $y * x \in \zeta_{\alpha}\left(\omega_{\alpha}\right)$, then $y \in \zeta_{\alpha}\left(\omega_{\alpha}\right)$ since $\zeta_{\alpha}\left(\omega_{\alpha}\right)$ is an ideal of $X$. Hence $y * x \notin \zeta_{\alpha}\left(\omega_{\alpha}\right)$. Similarly, if $x \in \zeta_{\beta}\left(\omega_{\beta}\right)$ and $y \notin \zeta_{\beta}\left(\omega_{\beta}\right)$, then $y * x \notin \zeta_{\beta}\left(\omega_{\beta}\right)$.

Conversely, let $\tilde{G}:=\left(\tilde{G}_{\alpha}, \tilde{G}_{\beta}\right)$ be a falling intuitionistic subalgebra of a $B C I$-algebra $X$ that satisfies the condition Equation (19). Then $\zeta_{\alpha}\left(\omega_{\alpha}\right)$ and $\zeta_{\beta}\left(\omega_{\beta}\right)$ are subalgebras of $X$ for all $\omega_{\alpha}, \omega_{\beta} \in \Gamma$. Hence 0 is contained in $\zeta_{\alpha}\left(\omega_{\alpha}\right)$ and $\zeta_{\beta}\left(\omega_{\beta}\right)$. Let $x, y, c, d \in X$ be such that $x * y \in \zeta_{\alpha}\left(\omega_{\alpha}\right)$, $y \in \zeta_{\alpha}\left(\omega_{\alpha}\right), c * d \in \zeta_{\beta}\left(\omega_{\beta}\right)$ and $d \in \zeta_{\beta}\left(\omega_{\beta}\right)$. If $x \notin \zeta_{\alpha}\left(\omega_{\alpha}\right)$ (resp., $c \notin \zeta_{\beta}\left(\omega_{\beta}\right)$ ), then $x * y \notin \zeta_{\alpha}\left(\omega_{\alpha}\right)$ (resp., $c * d \notin \zeta_{\beta}\left(\omega_{\beta}\right)$ ) by Equation (19). This is a contradiction, and so $x \in \zeta_{\alpha}\left(\omega_{\alpha}\right)$ and $c \in \zeta_{\beta}\left(\omega_{\beta}\right)$. Therefore $\tilde{G}:=\left(\tilde{G}_{\alpha}, \tilde{G}_{\beta}\right)$ is a falling intuitionistic ideal of $X$.

\section{Conclusions}

As a general concept of the fuzzy random set and fuzzy falling shadow, we have considered the concepts of intuitionistic random set and intuitionistic falling shadow. Using these generalized notions, we have introduced the notion of falling intuitionistic subalgebra and falling intuitionistic ideal of $B C K / B C I$-algebras. We have discussed relations between falling intuitionistic subalgebra and falling intuitionistic ideal, and have established a characterization of falling intuitionistic ideal.

Author Contributions: This paper is a result of common work of the authors in all aspects.

Funding: This research received no external funding.

Acknowledgments: The authors wish to thank the anonymous reviewers for their valuable comments and suggestions. The second author was supported under the framework of international cooperation program managed by the National Research Foundation of Korea (2017 K2A9A1A01092970, FY2017).

Conflicts of Interest: The authors declare no conflict of interest. 


\section{References}

1. Atanassov, K.T. Intuitionistic fuzzy sets. Fuzzy Sets Syst. 1986, 20, 87-96. [CrossRef]

2. Goodman, I.R. Fuzzy sets as equivalence classes of random sets. In Recent Developments in Fuzzy Sets and Possibility Theory; Yager, R., Ed.; Pergamon: New York, NY, USA, 1982; pp. 327-343.

3. Wang, P.Z.; Sanchez, E. Treating a fuzzy subset as a projectable random set. In Fuzzy Information and Decision; Gupta, M.M., Sanchez, E.; Eds.; Pergamon: New York, NY, USA, 1982; pp. 212-219.

4. Wang, P.Z. Fuzzy Sets and Falling Shadows of Random Sets; Beijing Normal Univ. Press: Beijing, China, 1985. (In Chinese)

5. Tan, S.K.; Wang, P.Z.; Lee, E.S. Fuzzy set operations based on the theory of falling shadows. J. Math. Anal. Appl. 1993, 174, 242-255. [CrossRef]

6. Tan, S.K.; Wang, P.Z.; Zhang, X.Z. Fuzzy inference relation based on the theory of falling shadows. Fuzzy Sets Syst. 1993, 53, 179-188. [CrossRef]

7. Jun, Y.B.; Park, C.H. Falling shadows applied to subalgebras and ideals of BCK/BCI-algebras. Honam Math. J. 2012, 34, 135-144. [CrossRef]

8. Jun, Y.B.; Kang, M.S. Fuzzy positive implicative ideals of BCK-algebras based on the theory of falling shadows. Comput. Math. Appl. 2011, 61, 62-67. [CrossRef]

9. Iséki, K. On BCI-algebras. Math. Semin. Notes 1980, 8, 125-130.

10. Iséki, K.; Tanaka, S. An introduction to the theory of BCK-algebras. Math. Jpn. 1978, 23, 1-26.

11. Huang, Y. BCI-Algebra; Science Press: Beijing, China, 2006.

12. Meng, J.; Jun, Y.B. BCK-Algebras; Kyungmoon Sa Co.: Seoul, Korea, 1994.

13. Jun, Y.B.; Kim, K.H. Intuitionistic fuzzy ideals of BCK-algebras. Int. J. Math. Math. Sci. 2000, 24, 839-849. [CrossRef]

14. Chen, Z.M.; Wang, H.X. On ideals in BCI-algebras. Math. Jpn. 1991, 36, 497-501.

(C) 2018 by the authors. Licensee MDPI, Basel, Switzerland. This article is an open access article distributed under the terms and conditions of the Creative Commons Attribution (CC BY) license (http:/ / creativecommons.org/licenses/by/4.0/). 